\title{
Diagnostic Thinking Process in Different Learning Environments
}

\author{
Or, P. P. L. ${ }^{1}$, So, H.C.F. ${ }^{1}$, Chen, J. ${ }^{1}$, Choi, M.M.T. ${ }^{1}$, Chan, C.K.M. ${ }^{2}$, Chan K.K. ${ }^{3}$, Leung \\ C.L.C. ${ }^{4}$, Chung, J.W.Y ${ }^{5}$ \\ Department of Nursing and Health Sciences, Tung Wah College, Hong Kong ${ }^{1}$ \\ Hong Kong Sport Institute, Hong Kong ${ }^{2}$ \\ The Nethersole School of Nursing, The Chinese University of Hong Kong ${ }^{3}$ \\ School of Nursing, Grantham Hospital, Hong Kong ${ }^{4}$ \\ Department of Health and Physical Education, The Hong Kong Institute of \\ Education, Hong Kong ${ }^{5}$
}

\begin{abstract}
The objective of this study was to explore the diagnostic thinking process among nursing students in a different teaching and learning environment. A quasi-experimental design was selected in this study because it can demonstrate the complexity of the clinical situation through a scenario with its descriptive nature. This study is likely to distinguish the difference from two learning environments in terms of learning styles among nursing students.
\end{abstract}

\section{Introduction}

Diagnostic thinking refers to the cognitive process, which result in the formation of a diagnosis. This thinking process includes consideration of diverse factors, including the patient's physical disability and impairment.

Diagnosing is the most critical element of the nursing process because it is the first clinical judgment made by nurses in the nursing process. Any mistake in diagnosing can severely affect the treatment process and even decrease the curing rate of patients.

Diagnosing remains uncertain for nursing students because diagnostic reasoning is not a commonplace in nursing education despite its importance. The learning style is a key determinant of the diagnostic thinking process. Salehi suggested that the stage of learning involving thinking and problem analysis [1]. Hospitals have a growing proportion of patients with complex health problem; at the same time, clinical learning environments are growing more unpredictable in quality due to nursing shortages and reductions in patients' lengths of stay [6].

Wong and Chung have examined the diagnostic thinking process among nursing students from pre-registration nursing programme with different learning environment [8]. The results showed that participants from the nursing school adopted backward reasoning strategies for decision-making whereas one third of the students from the university adopted forward reasoning strategies. Botti and Reeve suggested that academic ability determined the effectiveness of clinical decision-making process [9]. Therefore, the objective of this study was to explore the diagnostic thinking process among nursing students in a different teaching and learning environment [7].

\section{Method}

A quasi-experimental design with random sequence of scenarios was selected in this study because it can demonstrate the complexity of the clinical situation through a scenario with its descriptive nature, and also this can help to understand and interpret subjects' diagnostic thinking processes in a systematic approach. Biggs' Study Process Questionnaire (SPQ) was used to evaluate the learning style among subjects. The Biggs' study process has two components: motive and strategy. Motive is the meaning the student looks for in the learning task, and strategy is the way the student tackles the task. There are three common approaches to learning: surface, deep, and achieving. There are 42 items in the SPQ, and it produces scores categorizing students' study approach into 6 sub-scales, which include

$\begin{array}{ll}\text { i. } & \text { surface motive } \\ \text { ii. } & \text { deep motive } \\ \text { iii. } & \text { achieving motive } \\ \text { iv. } & \text { surface strategy } \\ \text { v. } & \text { deep strategy, and } \\ \text { vi. } & \text { achieving strategy. }\end{array}$

Each sub-scale contains 7 items. All items are scored in the same direction. The construct validity of the SPQ has been established from a large number of 
studies, most of which were summarized in Biggs [2] [3].

Three scenarios were used in this study by making use of the patient simulator, as they could reflect different levels of clinical complexity. The first scenario was a patient with a known specific problem; the second concerned a non-specific problem, while the third was an emergency condition. To ensure the ecological validity of the simulated scenarios, the data entered into the Patient Simulation System (PSS) were based on real clinical parameters. The PSS allows students to perform nursing procedures as if they were in a real clinical situation. They can listen to the simulator's heart sounds and breathing sounds, and can also feel the pulsation of the carotid and radial arteries. Students can talk to the simulator and it will respond verbally (this is because the instructor controlling the console acts as the simulator and responds to students via the microphone installed in the simulator). Apart from this, students can administer oxygen to the simulator and the gas sensor in the simulator can then produce the relevant physiological response, which will be shown on the computer screen. The use of a patient simulator in testing clinical performance has been documented, with high inter-rater reliability $($ kappa $=0.96)[4]$.

\subsection{Sampling}

Students, studying in their last year of Enrolled Nurse programme, were eligible. According to the similar study from Wong \& Chung, the estimated sample size of the study was set to 40, 20 from a college and 20 from a hospital-based nursing school [5].

\subsection{Procedure}

Ethics was approved by the Ethical Review Committee of the College. Subjects were informed to think aloud as they were performing a set of specified tasks and the whole process would be videotaped. Before the study, subjects were introduced to various functions of the simulator to reduce their anxieties and familiarize them with the environment. Subjects were asked to complete the SPQ and then asked to draw the sequence of the scenarios randomly. They were informed about the background of the scenarios, including the name and age of the patient, day and time of the event and a brief medical history. They were required identifying the differential diagnosis in the three simulated scenarios, and 5 minutes were allowed for each scenario. Upon completion of the task, they were interviewed and asked about their interpretation of the patient's condition, differential diagnosis and the problems that the patient might have. They could terminate the scenario at any time when they believed they have come to a diagnostic label. The simulation sessions were recorded and problem behavior graphs (PBGs) were drawn to determine their diagnostic thinking processes. A problem behavior graph is a method used to analyze and depict the thought processes made explicit when a subject thinks aloud as they solve a problem [10]. It is a collection of nodes that are linked together both horizontally and vertically. A horizontal node represents backward chaining strategies for decision-making. A vertical node represents forward chaining strategies i.e. data-driven. This also means that time spent working on the problem is reflected in both the horizontal and vertical dimension of the problem behavior graph.

\section{Results}

Forty-two subjects were randomly drawn from students in the last year of a pre-enrollment nursing programme in two learning institutions, 21 from a college and 21 from a hospital-based nursing school. Table 1 shows the learning styles of the students. Students from the nursing school and college had higher mean scores in using the deep approach. The distributions of the scores of students from college were less dispersed. They scored higher on surface motive, achieving motive and surface strategy.

Hospital-based training students had higher scores in deep motive.

Table 1. Pattern of study approaches of the subjects

\begin{tabular}{lll}
\hline & \multicolumn{2}{l}{ Mean score } \\
\cline { 2 - 3 } Approach & College & Nursing school \\
\hline Surface & 47.76 & 43.10 \\
Deep & 49.86 & 51.86 \\
Achieving & 43.71 & 43.33 \\
\hline
\end{tabular}

The process of diagnostic thinking was videotaped.

Transcription and theming and problem behavior graphs (PBGs) were drawn to determine their diagnostic thinking processes. Subjects from the college have had more vertical $(53 \%)$ patterns in the PBGs than subjects from nursing school (47\%). While subjects from nursing school have had more horizontal $(52 \%)$ patterns in the PBGs than subjects from the college $(48 \%)$.

\section{Discussion}

Students from hospital-based training school adopted a deep strategy more than students from other nursing training programmes. They were characteristically neat and systematic. College students had higher scores in surface strategy. Surface-achievers systematically recite selected details to obtain high grades; excel in rote learning. Surface and Deep approaches are mutually exclusive. 
Comparing their reasoning patterns, more than half of the college students adopted forward chaining strategies (vertical), i.e. data-driven. They used open-ended exploration of data, to arrive at conclusions. In contrast, students from hospital-based training school adopted more backward chaining (horizontal) strategies for decision-making. They formulated hypotheses and tested them by collecting data. Thus, a hypothesis-driven strategy was employed. When using hypothesis-driven strategies, the clinical decision was always context-dependent.

The graduation credit requirement was higher in the nursing programme conducted by the college than in the hospital-based training school. Thus, in the former, the students' workload was perceived as heavy. Time pressures and, stress from assignments may influence which learning approach a student develops. In cases of extreme pressure, students will attempt to cope by adopting a surface approach to learning.

Findings of this study have highlighted the variations in decision strategies among students in different learning environment. In particular, the use of patient simulation is useful in teaching and learning activities. Hospitals cannot afford to have students practicing on patients without adequate training and preparation beforehand. More importantly, the use of patient simulation can provide a safe environment for students to acquire the necessary clinical competency and allow them unlimited practice. With these different approaches, students are encouraged to acquire the necessary knowledge and skills, and are allowed to practice their diagnostic thinking in a controlled environment. This is important in preparing nursing students to be competent practitioners. Area for further work include investigate the association between the types of scenario and diagnostic thinking process.

\section{Conclusion}

Diagnosing is a critical element in nursing training. Stage of learning is a key determinant of diagnostic reasoning process. This study is likely to distinguish the difference from two learning environments in terms of study approach among nursing students. To this end, it can be suggested a variety of methods of teaching so as to revisit the current nursing education in Hong Kong.

\section{References}

[1] Salehi, Sh. (2007). Nursing students' preferred learning style. Journal of Medical Education 11, 85-89.

[2] Biggs J. (1987). Student Approaches to Learning and Studying. Australian Council for Educational Research, Melbourne.
[3] Biggs J. (1992). Why and How do Hong Kong Students Learn? Using the Learning and Study Process Questionnaire. University of Hong Kong, Hong Kong.

[4] Devitt J.H., Kurrek M.M., Cohen M.M., Fish K., Fish P., Murphy P.M. \& Szalai J.P. (1997). Testing the raters: inter-rater reliability of standardized anesthesia simulator performance. Canadian Journal of Anaesthesiology 44(9), 924-928.

[5] Wong K.S.T. \& Chung J.W.Y. (2002). Diagnostic reasoning processes using patient simulation in different learning environments. Journal of Clinical Nursing 11, 65-72.

[6] Bright, D., Walker, W., Bion, J., (2004). Clinical review: outreach-a strategy for improving the care of the acutely ill hospitalized patient. Critical Care 8, 33-40.

[7] Levett-Jones, T., \& Bourgeois, S. (2007). The clinical placement: An essential guide for nursing students. Sydney: Elsevier.

[8] Wong, K.S., \& Chung, J.W.Y. (2002). Diagnostic reasoning processes using patient simulation in different learning environments. Journal of Clinical Nursing 11, 65-72.

[9] Botti, M. \& Reeve, R. (2003). Role of knowledge and ability in student nurses' clinical decision-making. Nursing and Health Science, 5, 39-49.

[10] Dawson, M.R.W. \& Medler, D.A. (2010) Problem Behaviour Graph University of Alberta. Retrieved on December 2013 from http://www.bcp.psych.ualberta.ca/ mike/Pearl_Street/Dictio nary/contents/P/pbg.html 